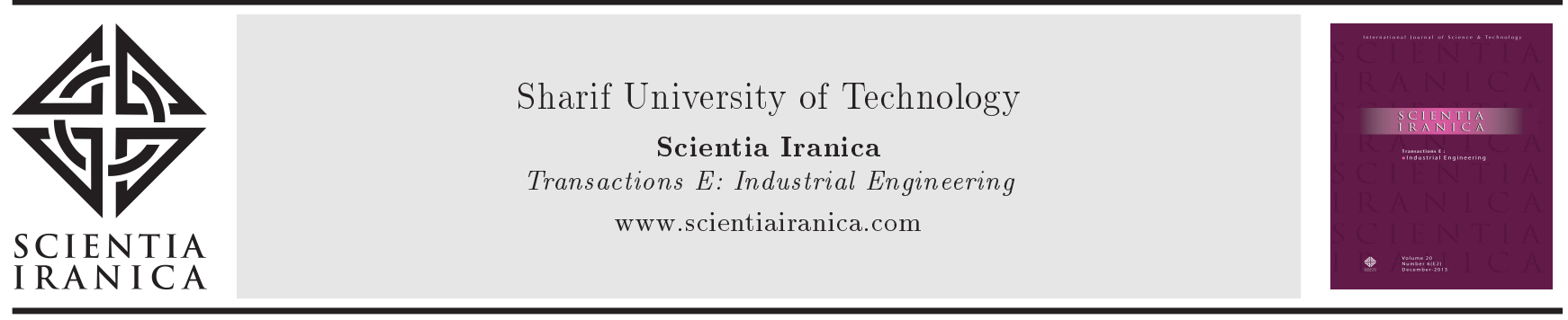

\title{
A two-stage stochastic programming model for value-based supply chain network design
}

\author{
H. Badri ${ }^{a}$, S.M.T. Fatemi Ghomi ${ }^{a, *}$ and T.H. Hejazi ${ }^{b}$ \\ a. Department of Industrial Engineering, Amirkabir University of Technology, 424 Hafez Avenue, Tehran, P.O. Box 1591634311, \\ Iran. \\ b. Young Researchers and Elite Club, Qazvin Branch, Islamic Azad University, Qazvin, Iran.
}

Received 6 May 2014; received in revised form 18 November 2014; accepted 10 March 2014

\section{KEYWORDS}

Supply chain network design;

Value-based

management;

Two-stage stochastic

programming;

Correlated

parameters.

\begin{abstract}
Nowadays, Value-based Supply Chain Management (VbSCM) is considered a resource of competitive advantages, and companies with long term strategic plans find the $\mathrm{VbSCM}$ an effective factor in sustainability. In this context, supply chain network design has a significant impact on all value drivers (i.e. sales, supply chain costs, fixed assets and working capital). This paper proposes a stochastic mixed integer linear programming model for a value-based supply chain network design in which decisions on physical flow (raw materials and finished products) and financial flow are integrated. The proposed model is designed for a four-echelon, multi-commodity, multi-period supply chain, and it maximizes the value of the company, based on the economic value-added concept, by making some strategic and tactical decisions affecting the value drivers. Furthermore, a scenario-based two-stage stochastic programming model is developed with a scenario generation method based on Nataf transformation. Also, a computational analysis is undertaken to illustrate the performance of the proposed approach.
\end{abstract}

(C) 2016 Sharif University of Technology. All rights reserved.

\section{Introduction}

In the past decade, the concept of Value-based Management $(\mathrm{VbM})$, whose core objective is to increase the value of a firm, has been applied to supply chain management and is known as Value-based Supply Chain Management (VbSCM) [1-4]. In this approach, the value of a company is calculated by its ability to create future cash flow, which, in turn, is driven by profitability, capital efficiency and cost of capital $[5,6]$. Furthermore, profitability, capital efficiency and cost of capital are affected by management decisions of operations, investment and financing $[1,7]$.

In Supply Chain Network Design (SCND), configuration of a supply chain requires different strategic and tactical decisions, such as facility location,

* Corresponding author. Tel.: +98 2164545381 ;

E-mail address: fatemi@aut.ac.ir (S.M.T. Fatemi Ghomi) technology selection and production, and distribution planning. These decisions have a long-lasting effect on the survivability of a firm. Furthermore, most of these strategic and tactical decisions are highly correlated with financial flow in the supply chain.

In classical supply chain network design, it is usually assumed that the value of a company is affected only by its sales or costs [8-10]. Based on this assumption, other important value drivers, such as working capital and fixed assets, are ignored in the design and planning process. But, based on the VbSCM approach, all value drivers are taken into consideration. From this point of view, supply chain management influences company value via four financial drivers, including sales, cost, working capital and fixed assets. All decisions made by the management system in financing, investing and operating affect the value drivers.

In recent years, many conceptual frameworks have 
Table 1. Structure of the stochastic models in supply chain network design.

\begin{tabular}{|c|c|c|c|c|c|c|c|c|c|}
\hline \multirow{2}{*}{ Paper } & \multirow{2}{*}{$\begin{array}{l}\text { SCM } \\
\text { level }\end{array}$} & \multirow{2}{*}{$\begin{array}{c}\text { Location } \\
\text { level }\end{array}$} & \multicolumn{2}{|c|}{ Production stage } & \multicolumn{2}{|c|}{ Time period } & \multicolumn{2}{|c|}{ Final products } & \multirow{2}{*}{$\begin{array}{l}\text { Objective } \\
\text { function }\end{array}$} \\
\hline & & & Single & Multiple & Single & Multiple & Single & Multiple & \\
\hline Aghezzaf [8] & 2 & 1 & $\checkmark$ & & & $\checkmark$ & $\checkmark$ & & $\mathrm{TC}^{\mathrm{a}}$ \\
\hline Ambrosino & & & & & & & & & \\
\hline $\begin{array}{c}\text { and Scutell } \\
{[9]}\end{array}$ & $\geq 3$ & $\geq 3$ & $\checkmark$ & & & $\checkmark$ & $\checkmark$ & & $\mathrm{TC}$ \\
\hline $\begin{array}{c}\text { Chan et al. } \\
{[18]}\end{array}$ & 1 & 1 & $\checkmark$ & & $\checkmark$ & & $\checkmark$ & & $\mathrm{TC}$ \\
\hline $\begin{array}{c}\text { Melkote and } \\
\text { Daskin [19] }\end{array}$ & 2 & 1 & $\checkmark$ & & $\checkmark$ & & $\checkmark$ & & $\mathrm{TC}$ \\
\hline $\begin{array}{l}\text { Guillén } \\
\text { et al. [16] }\end{array}$ & 2 & 2 & $\checkmark$ & & $\checkmark$ & & & $\checkmark$ & MO \\
\hline Hwang [20] & 2 & 1 & $\checkmark$ & & $\checkmark$ & & $\checkmark$ & & $\mathrm{TC}$ \\
\hline $\begin{array}{l}\text { Lieckens and } \\
\text { Vandaele [21] }\end{array}$ & 2 & 1 & $\checkmark$ & & $\checkmark$ & & $\checkmark$ & & $\mathrm{TNP}^{\mathrm{b}}$ \\
\hline $\begin{array}{l}\text { Lowe } \\
\text { et al. [22] }\end{array}$ & 1 & 1 & $\checkmark$ & & $\checkmark$ & & $\checkmark$ & & $\mathrm{TC}$ \\
\hline $\begin{array}{l}\text { Miranda and } \\
\text { Garrido [23] }\end{array}$ & 2 & 1 & $\checkmark$ & & $\checkmark$ & & $\checkmark$ & & $\mathrm{TC}$ \\
\hline $\begin{array}{l}\text { Miranda and } \\
\text { Garrido [24] }\end{array}$ & 2 & 1 & $\checkmark$ & & $\checkmark$ & & $\checkmark$ & & $\mathrm{TC}$ \\
\hline $\begin{array}{l}\text { Pishvaee and } \\
\text { Torabi [10] }\end{array}$ & $\geq 3$ & $\geq 3$ & $\checkmark$ & & & $\checkmark$ & $\checkmark$ & & $\mathrm{TC}$ \\
\hline $\begin{array}{c}\text { Sabri and } \\
\text { Beamon }[17]\end{array}$ & $\geq 3$ & 2 & $\checkmark$ & & $\checkmark$ & & & $\checkmark$ & $\mathrm{MO}^{\mathrm{c}}$ \\
\hline $\begin{array}{l}\text { Shen and } \\
\text { Qi [25] }\end{array}$ & 1 & 1 & $\checkmark$ & & $\checkmark$ & & $\checkmark$ & & $\mathrm{TC}$ \\
\hline $\begin{array}{l}\text { Snyder } \\
\text { et al. [26] }\end{array}$ & 1 & 1 & $\checkmark$ & & $\checkmark$ & & $\checkmark$ & & $\mathrm{TC}$ \\
\hline $\begin{array}{l}\text { Ommeren } \\
\text { et al. [27] }\end{array}$ & 2 & 1 & $\checkmark$ & & $\checkmark$ & & $\checkmark$ & & $\mathrm{TC}$ \\
\hline This paper & $\geq 3$ & 2 & $\checkmark$ & & & $\checkmark$ & & $\checkmark$ & EVA \\
\hline
\end{tabular}

${ }^{\mathrm{a}} \mathrm{TC}$ : Total Cost; ${ }^{\mathrm{b}} \mathrm{TNP}$ : Total Net Profit; ${ }^{\mathrm{c}} \mathrm{MO}$ : Multiple Objective.

been proposed for $\mathrm{VbSCM}$ in which some elements, such as revenue growth, operating costs or operating capital, are considered value drivers [11-14]. In comparison to these conceptual models for VbSCM, a limited number of endeavours have been undertaken concentrating on quantitative models $[1,3,15]$.

In the literature of SCND, there are several stochastic models developed to help managers in configuration of their supply chain. Most of the proposed stochastic models for SCND are categorized as static models in which decisions are made for a single period. In contrast, there are few multiple period models developed for SCND [8-10]. Production in all reviewed papers is supposed to be done in a single stage. Most of the reviewed papers have considered a single product, but a few have considered multiple products. When it comes to the objective function, total cost is the most prevalent objective function in the reviewed papers $[16,17]$. Also, some papers considered multiple objectives in their proposed model. Table 1 shows the structure of the reviewed stochastic models in the field of SCND.

Production planning, inventory management and capacity planning are the most prevalent decisions in the stochastic models of SCND. Also, routing has been considered in a few papers $[9,18,20]$. From four value drivers proposed by Rappaport [7], supply chain costs have been considered in all reviewed models; but none of the reviewed papers have considered three remaining value drivers, i.e. sales growth, working capital and fixed assets. Table 2 illustrates decisions in the reviewed models in the field of SCND.

This paper proposes a two-stage programming model for value-based supply chain network design. 
Table 2. Decisions in the stochastic models in supply chain network design.

\begin{tabular}{|c|c|c|c|c|c|c|}
\hline \multirow[b]{2}{*}{ Paper } & \multicolumn{6}{|c|}{ Decisions } \\
\hline & Procurement & $\begin{array}{c}\text { Production } \\
\text { planning }\end{array}$ & $\begin{array}{c}\text { Inventory } \\
\text { management }\end{array}$ & $\begin{array}{l}\text { Capacity } \\
\text { planning }\end{array}$ & $\begin{array}{c}\text { Finance, asset } \\
\text { management, } \\
\text { and pricing }\end{array}$ & Routing \\
\hline Aghezzaf [8] & & & $\checkmark$ & $\checkmark$ & & \\
\hline $\begin{array}{c}\text { Ambrosino and } \\
\text { Scutell [9] }\end{array}$ & & & $\checkmark$ & & & $\checkmark$ \\
\hline Chan et al. [18] & & & & & & $\checkmark$ \\
\hline $\begin{array}{l}\text { Melkote and } \\
\text { Daskin [19] }\end{array}$ & & & $\checkmark$ & & & \\
\hline Guillén et al. [16] & & $\checkmark$ & $\checkmark$ & $\checkmark$ & & \\
\hline Hwang [20] & & & & & & $\checkmark$ \\
\hline $\begin{array}{l}\text { Lieckens and } \\
\text { Vandaele [21] }\end{array}$ & & & $\checkmark$ & $\checkmark$ & & \\
\hline $\begin{array}{l}\text { Lowe } \\
\text { et al. [22] }\end{array}$ & & $\checkmark$ & & $\checkmark$ & & \\
\hline $\begin{array}{l}\text { Miranda and } \\
\text { Garrido [23] }\end{array}$ & & & $\checkmark$ & & & \\
\hline $\begin{array}{l}\text { Miranda and } \\
\text { Garrido [24] }\end{array}$ & & & $\checkmark$ & & & \\
\hline $\begin{array}{l}\text { Pishvaee and } \\
\text { Torabi [10] }\end{array}$ & & $\checkmark$ & & & & \\
\hline $\begin{array}{c}\text { Sabri and } \\
\text { Beamon }[17]\end{array}$ & & $\checkmark$ & & & & \\
\hline $\begin{array}{l}\text { Shen and } \\
\text { Qi }[25]\end{array}$ & & & $\checkmark$ & & & \\
\hline $\begin{array}{l}\text { Snyder } \\
\text { et al. [26] }\end{array}$ & & & $\checkmark$ & & & \\
\hline $\begin{array}{l}\text { Ommeren } \\
\text { et al. [27] }\end{array}$ & & & $\checkmark$ & $\checkmark$ & & \\
\hline This paper & $\checkmark$ & $\checkmark$ & $\checkmark$ & $\checkmark$ & $\checkmark$ & \\
\hline
\end{tabular}

The proposed model considers both strategic and tactical decisions in the supply chain. The objective of the proposed model is to maximize the value of the company during the planning horizon. For this purpose, four value drivers are considered: supply chain costs, sales growth, working capital, and fixed assets. The proposed model covers decisions in operations, financing and debt management.

Furthermore, in the proposed model, a fourechelon supply chain is considered, including suppliers, production facilities, distribution centers and customer zones. All products are manufactured in a single stage. Also, two kinds of warehouse are considered, a warehouse inside the production facilities for storing raw materials, and a warehouse in distribution centers for the finished products. In this network, finished products should be shipped to the customer zones from the distribution centers, and they cannot be transported directly from the production facilities to the customer zones. Figure 1 illustrates the structure

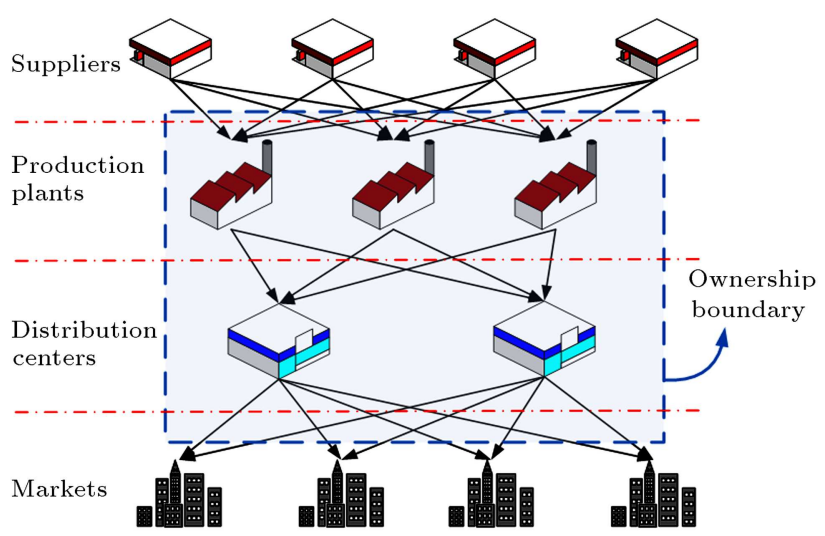

Figure 1. Structure of the considered supply chain.

of the supply chain considered in the proposed model. It is easily seen in this network that the company owns all production facilities and distribution centers.

The most important assumptions in the proposed model are as follows: 
- Customer demand and associated prices are stochastic parameters with correlated behavior;

- The total number of distributed products in a market cannot violate the predicted demand for that time period;

- Short term debt is considered the main source of financing;

- An opened facility cannot be closed during the planning period;

- The capacity of manufacturing facilities is determined by the amount of installed manufacturing equipment;

- Transfers are not permitted between warehouses;

- All manufactured products are distributed through distribution centers;

- Depreciation is only considered for the manufacturing equipment;

- Safety stock is required for both raw materials and finished products;

- Raw materials are stored in manufacturing facilities, and finished products are stored in distribution centers.

Some of the most important decisions in the proposed model are as follows:

- Location and establishment time of facilities (production plant, warehouse);

- Amount of manufacturing equipment to be installed in each facility in each period;

- Total amount of each type of raw material to be supplied by each potential supplier;

- Total number of each product to be produced in each manufacturing facility;

- Total number of each finished product transported from each manufacturing facility to each distribution center;

- Financing decisions.

The remaining part of the paper is organized as follows: The next section includes the proposed mathematical programming model. Section 3 presents the two-stage stochastic programming approach. Computational analysis is presented in Section 4, and finally, conclusions are drawn is Section 5 .

\section{Mathematical programming model}

This section proposes a Mixed Integer Linear Programming (MILP) model for the VbSCND in a multiple echelon, multiple commodity, and multiple period supply chain.

\section{Notations}

\section{Sets}

$\mathcal{T}\left(t \in \mathcal{T}, t=t_{0}, \ldots, T\right)(\tau \subset \mathcal{T})$ Set of time periods;

$\mathcal{S}(s \in \mathcal{S}) \quad$ Set of suppliers;

$I(i \epsilon I) \quad$ Set of production plants and distribution centers;

$\mathcal{M}(\mathcal{M} \subset i) \quad$ Set of production plants;

$\mathcal{W}(\mathcal{W} \subset i) \quad$ Set of distribution centers;

$\mathcal{C}(c \in \mathcal{C}) \quad$ Set of customers;

$P(p \in P) \quad$ Set of products (raw material and finished products);

$P_{r}\left(P_{r} \subset P\right) \quad$ Set of raw materials;

$P_{f}\left(P_{f} \subset P\right)$ Set of finished products.

\section{Parameters}

$i^{\text {wacc }} \quad$ Weighted average cost of capital;

$i^{t r} \quad$ Tax rate;

$i^{d} \quad$ Interest rate for short term debts;

$\mathrm{BM} \quad$ A very large number;

$D_{p, c}^{t} \quad$ Demand of finished product $p$ in customer zone $c$;

$\mathrm{COP}_{p}^{t} \quad$ Cost price;

$b v^{t} \quad$ Net book value at the end of lifetime period $t$;

$s v \quad$ Salvage value of technical equipment;

$\mathcal{P} V C_{p, i} \quad$ Production variable cost in facility $i$;

$\mathrm{SVM}_{p, i} \quad$ Storage variable cost for raw materials in production facilities;

$\mathrm{SVW}_{p, i} \quad$ Storage variable cost for finished products in distribution centers;

CAP Capacity of each unit of manufacturing equipment;

$C o_{i}^{t} \quad$ Fixed cost for opening facility $i$ in period $t$;

$C U_{i} \quad$ Fixed cost of operating facility $i$;

$B_{p, r} \quad$ Quantity of raw material, $r$, necessary to produce a unit of product $p$ (bill of materials);

$\operatorname{ICAP}_{i} \quad$ Capacity for storage at production facility/distribution center;

$\mathrm{IS}_{p} \quad$ Space occupied by unit raw material or finished product;

MSTD Maximum available short term debts in each period;

$\mathcal{P} \mathcal{R}_{p, c}^{t} \quad$ Price of a unit of finished product $p$;

$\mathcal{P} S_{p}^{t} \quad$ Price of a unit of raw material $p$;

$\mathrm{TC}_{p, s, i} \quad$ Transportation variable cost for raw material $p$ to be transported from supplier $s$ to production facility $i$; 
$\mathrm{TC}_{p, i, i^{\prime}} \quad$ Transportation variable cost for finished product $p$ to be transported from production facility $i$ to distribution center $i^{\prime}$;

$\mathrm{TC}_{p, i, c} \quad$ Transportation variable cost for finished product $p$ to be transported from distribution center $i$ to customer zone $c$;

$A_{i, j} \quad$ Number of deliveries from node $i$ to node $j$ in one period;

ICASH Position of cash at period $t_{0}$;

$I h_{p} \quad$ Position of inventory at period $t_{0}$;

$\alpha_{p} \quad$ Coefficient for safety stock of raw material $p$;

$\beta_{p} \quad$ Coefficient for safety stock of finished product $p$;

$\mathrm{SH}^{t} \quad$ Stockholder share.

\section{Decision variables}

EVA Economic Value Added;

NOPAT Net Operating Profit After Tax;

NOA Net Operating Assets;

$\mathrm{TCM}^{t} \quad$ Total Contribution Margin in period $t$;

$\mathrm{DEP}^{t} \quad$ Depreciation and capital loss of disinvestments in period $t$;

FA Fixed Assets;

$\mathrm{MEQ}_{i}^{t, \tau} \quad$ Stock of manufacturing equipment at location $i$ at the end of period $t$ acquired in period $\tau$;

$h m_{P_{r}, i}^{t} \quad$ Inventory of raw material $P_{r}$ in manufacturing facility $i$ at the end of period $t$;

$h w_{P_{f}, j}^{t} \quad$ Inventory of product $P_{f}$ in distribution center $j$ at the end of period $t$;

$\mathrm{AR}^{t} \quad$ Account Receivable;

$\mathrm{AP}^{t} \quad$ Account Payable;

$\mathrm{CASH}^{t} \quad$ Cash in period $t$;

$\mathrm{CFO}^{t} \quad$ Cash Flow from Operations;

$\mathrm{CFI}^{t} \quad$ Cash Flow from open Items;

CFS $^{t}$ Cash Flow from Short term financial investment;

$\mathrm{CFA}^{t} \quad$ Cash Flow from fixed Assets;

$\mathrm{CFD}^{t} \quad$ Cash Flow from Debt management;

$\mathrm{CA}^{t} \quad$ Current net Assets at the end of period $t$;

$f_{P_{r}, s, i}^{t} \quad$ Flow of raw material from supplier $s$ to manufacturing facility $i$ at the beginning of period $t$;

$$
\begin{array}{ll}
f_{P_{f}, i, j}^{t} & \begin{array}{l}
\text { Flow of raw material from } \\
\text { manufacturing facility } i \text { to distribution } \\
\\
\text { center } j \text { at the beginning of period } t ;
\end{array} \\
f_{P_{f}, j, c}^{t} & \text { Flow of raw material from distribution } \\
& \text { center } j \text { to customer } c \text { at the beginning } \\
& \text { of period } t ; \\
& \text { Binary variable, } 1, \text { if a facility } \\
& \text { (production facility / distribution } \\
& \text { center is open in location } i \text { in period } \\
& t, \text { otherwise, } 0 .
\end{array}
$$

\section{Objective function}

Shareholder value is created when earnings exceed total costs of invested capital [7]. Among different metrics, Economic Value Added (EVA) is the most prevalent metric of value-based performance $[1,3,15]$. Therefore, the objective function in the proposed model is to maximize the EVA over the time periods computed by Net Operating Profit After Tax (NOPAT) in period $t$ minus total costs of invested capital in Net Operating Assets (NOA) at the end of the previous period, adjusted by the weighted average cost of capital ( $\left.i^{\text {wacc }}\right)$ [28]. Eq. (1) shows the objective function in which economic value added is maximized:

$$
\begin{aligned}
& \text { Maximize EVA }=\sum_{t=t_{0}}^{T}\left(\mathrm{TCM}^{t}-\mathrm{DEP}^{t}\right) \cdot\left(1-i^{t r}\right) \\
& -\sum_{t=t_{0}}^{T}\left(f a^{t-1}+\sum_{i \in \mathcal{M}} \sum_{\tau=t_{0}}^{\tau=t-1} \mathrm{MEQ}_{i}^{t, \tau} \cdot\left(b v^{t-1-\tau}+s v\right)\right. \\
& \left.+\mathrm{CA}^{t-1}\right) \cdot i^{\text {wacc }} .
\end{aligned}
$$

\section{Constraints}

Eqs. (2)-(6) define elements of the objective function. In Eq. (2), depreciation of the manufacturing equipment is calculated and invested capital in fixed assets is calculated in Eq. (3). Eq. (4) is related to current assets, including inventories, accounts receivable and cash, minus accounts payable. In Eq. (5), the total contribution margin is calculated by subtracting total costs from total sales. Cost elements in this equation are fixed cost of operating facilities, supply variable cost, production variable cost, storage variable cost for raw materials, storage variable cost for finished products, and transportation variable cost between supplier and production facilities, between production facilities and distribution centers and between distribution centers and customer zones.

$$
\mathrm{DEP}^{t}=\sum_{i \in \mathcal{M}} \sum_{\tau=t_{0}}^{t-1} \mathrm{MEQ}_{i}^{t, \tau} \cdot\left(b v^{t-1-\tau}-b v^{t-\tau}\right) \quad \forall t \epsilon \mathcal{T},
$$




$$
\begin{aligned}
\mathrm{FA}^{t} & =\sum_{\forall i \in \mathcal{M} \cup \mathcal{W}} C o_{i}^{t} \cdot x_{i}^{t} \quad \forall t \epsilon \mathcal{T} \\
\mathrm{CA}^{t} & =\sum_{p \in P_{f}} \sum_{i \in \mathcal{W}} \mathrm{COP}_{p}^{t} \cdot h_{p, i}^{t}+\mathrm{AR}^{t}-\mathrm{AP}^{t}+\mathrm{CASH}^{t}
\end{aligned}
$$

$\forall t \epsilon \mathcal{T}$

$$
\begin{aligned}
& \mathrm{TCM}^{t}=\sum_{p \in P_{f}} \sum_{i \in \mathcal{W}} \sum_{c \in \mathcal{C}} \mathcal{P} \mathcal{R}_{p, c}^{t} \cdot f_{p, i, c}^{t}-\sum_{i \in \mathcal{M} \cup \mathcal{W}} \mathrm{CU}_{i} \cdot x_{i}^{t} \\
& -\sum_{p \in P_{r}} \sum_{s \in S} \sum_{i \in \mathcal{M}} \mathcal{P} S_{p}^{t} \cdot f_{p, s, i}^{t} \\
& -\sum_{p \in P_{f}} \sum_{i \in \mathcal{M}} \sum_{i^{\prime} \in \mathcal{W}} \mathcal{P V C}_{p, i} \cdot f_{p, i, i^{\prime}}^{t} \\
& -\sum_{p \in P_{f}} \sum_{i \in \mathcal{W}} \operatorname{SVM}_{p, i} \cdot\left(h w_{p, j}^{t}+\sum_{i^{\prime} \in \mathcal{M}} \frac{1}{2 A_{i^{\prime}, i}} \cdot f_{p, i^{\prime}, i}^{t}\right) \\
& -\sum_{p \in P_{r}} \sum_{i \in \mathcal{M}} \mathrm{SVW}_{p, i} \cdot\left(h m_{p, i}^{t}+\sum_{s \in S} \frac{1}{2 A_{s, i}} \cdot f_{p, s, i}^{t}\right) \\
& -\sum_{p \in P_{r}} \sum_{s \in S} \sum_{i \in \mathcal{M}} \mathrm{TC}_{p, s, i} \cdot f_{p, s, i}^{t} \\
& -\sum_{p \in P_{f}} \sum_{i \in \mathcal{M}} \sum_{i^{\prime} \in \mathcal{W}} \mathrm{TC}_{p, i, i^{\prime}} \cdot f_{p, i, i^{\prime}}^{t} \\
& -\sum_{p \in P_{f}} \sum_{i \in \mathcal{W}} \sum_{c \in \mathcal{C}} \mathrm{TC}_{p, i, c} \cdot f_{p, i, c}^{t} \\
& +\sum_{p \in P_{r}} \sum_{i \in \mathcal{M}} \mathcal{P} S_{p}^{t} \cdot\left(h m_{p, i}^{t}-h m_{p, i}^{t-1}\right) \\
& +\sum_{p \in P_{f}} \sum_{i \in \mathcal{W}} \mathcal{P} \mathcal{R}_{p, c}^{t} \cdot\left(h w_{p, i}^{t}-h w_{p, i}^{t-1}\right) \\
& \forall t \in \mathcal{T} \text {. }
\end{aligned}
$$

Eqs. (6) and (7) define the position of accounts receivable and accounts payable in each period, respectively:

$$
\sum_{p \in P_{f}} \sum_{i \in \mathcal{W}} \sum_{c \in \mathcal{C}} \mathcal{P} \mathcal{R}_{p, c}^{t} \cdot f_{p, i, c}^{t}-\mathrm{AR}^{t}=0
$$

$\forall t \epsilon \mathcal{T}$

$$
\sum_{p \in P_{f}} \sum_{i \in \mathcal{W}} \sum_{c \in \mathcal{C}} \operatorname{COP}_{p}^{t} \cdot f_{p, i, c}^{t}-\mathrm{AP}^{t}=0
$$

$\forall t \epsilon \mathcal{T}$

Eq. (8) is to ensure the equilibrium of financial flow in the supply chain in each period:

$$
\begin{aligned}
& \mathrm{CASH}^{t}= \mathrm{CASH}^{t-1}-\mathrm{CFO}^{t}-\mathrm{SH}^{t}+\mathrm{CFI}^{t} \\
&+\mathrm{CFS}^{t}+\mathrm{CFA}^{t}+\mathrm{CFD}^{t} \\
& \forall t \epsilon\left\{t_{0}+1, \cdots, T\right\} .
\end{aligned}
$$

In Eq. (9), operating cash flow in each period is calculated:

$$
\begin{aligned}
& \mathrm{CFO}^{t}=\sum_{i \in \mathcal{M} \mathcal{W}^{\mathcal{W}}} \mathrm{CU}_{i} \cdot x_{i}^{t}+\sum_{p \in P_{r}} \sum_{s \in S} \sum_{i \in \mathcal{M}} \mathcal{P} S_{p}^{t} \cdot f_{p, s, i}^{t} \\
& +\sum_{p \in P_{f}} \sum_{i \in \mathcal{M}} \sum_{i^{\prime} \in \mathcal{W}} \mathcal{P V C}_{p, i} \cdot f_{p, i, i^{\prime}}^{t} \\
& +\sum_{p \in P_{f}} \sum_{i \in \mathcal{W}} \mathrm{SVM}_{p, i} \cdot\left(h w_{p, i}^{t}+\sum_{i^{\prime} \in \mathcal{M}} \frac{1}{2 A_{i^{\prime}, i}} \cdot f_{p, i^{\prime}, i}^{t}\right) \\
& +\sum_{p \in P_{r}} \sum_{i \in \mathcal{M}} \mathrm{SVW}_{p, i} \cdot\left(h m_{p, i}^{t}+\sum_{s \in S} \frac{1}{2 A_{s, i}} \cdot f_{p, s, i}^{t}\right) \\
& +\sum_{p \in P_{r}} \sum_{s \in S} \sum_{i \in \mathcal{M}} \mathrm{TC}_{p, s, i} \cdot f_{p, s, i}^{t} \\
& +\sum_{p \in P_{f}} \sum_{i \in \mathcal{M}} \sum_{i^{\prime} \in \mathcal{W}} \mathrm{TC}_{p, i, i^{\prime}} \cdot f_{p, i, i^{\prime}}^{t} \\
& +\sum_{p \in P_{f}} \sum_{i^{\prime} \in \mathcal{W}} \sum_{c \in \mathcal{C}} \mathrm{TC}_{p, i^{\prime}, c} \cdot f_{p, i^{\prime}, c}^{t} \\
& +\sum_{\tau=t_{0}}^{t-1} \sum_{i \in \mathcal{M}} \sum_{i^{\prime} \in \mathcal{M}} \mathrm{RLC}_{i, i^{\prime}} \cdot R L_{i, i^{\prime}}^{t, \tau}=0 \\
& \\
& +
\end{aligned}
$$

Eq. (10) defines cash flow from open items, which is the amount of accounts receivable minus the amount of accounts payable in the previous period:

$$
\begin{gathered}
\mathrm{CFI}^{t}=\mathrm{AR}^{t-1}-\mathrm{AP}^{t-1} \\
\forall t \epsilon\left\{t_{0}+1, \cdots, T\right\} .
\end{gathered}
$$

Cash flow from financing, based on short term debt, is calculated in Eq. (11). In Eq. (12) the amount of short term debt borrowed in each period is restricted to the maximum debts available:

$$
\begin{aligned}
& \mathrm{CFS}^{t}=\mathrm{STD}^{t-1} \cdot\left(1+i^{d}\right)-\mathrm{STD}^{t} \\
& \forall t \epsilon\left\{t_{0}+1, \cdots, T\right\}, \\
& \mathrm{STD}^{t} \leq \mathrm{MSTD} \quad \forall t \epsilon \mathcal{T} .
\end{aligned}
$$

Cash flow from fixed assets is calculated in Eq. (13): 


$$
\begin{aligned}
& \mathrm{CFA}^{t}=-\sum_{i \in \mathcal{M}} \mathrm{MEQ}_{i}^{t, t} \cdot\left(b v_{0}+s v\right) \\
&-\sum_{i \in \mathcal{M} \cup \mathcal{W}} C o_{i}^{t} \cdot\left(x_{i}^{t}-x_{i}^{t-1}\right) \\
& \forall t \epsilon\left\{t_{0}+1, \cdots, T\right\} .
\end{aligned}
$$

Constraints (14)-(16) are related to the equilibrium of flows of raw materials and finished products in production facilities and distribution centers, respectively. The quantity of finished product stored at the beginning of the current period, plus the total quantity of the same product delivered to the distribution center during the current period, should be equal to the quantity of that product transported to customer zones plus the quantity stored at the beginning of the next period. Also, the quantity of raw material stored at the beginning of the current period, plus the total quantity of the same raw material delivered to a production facility during the current period, should be equal to the quantity of that raw material used to manufacture products during the current period plus the quantity stored at the beginning of the next period.

$$
\begin{aligned}
& h w_{p, i^{\prime}}^{t-1}+\sum_{i \in \mathcal{M}} f_{p, i, i^{\prime}}^{t}-\sum_{c \in \mathcal{C}} f_{p, i^{\prime}, c}^{t}-h w_{p, i^{\prime}}^{t}=0 \\
& \forall i^{\prime} \in \mathcal{W}, \forall p \in P_{f}, \forall t \epsilon\left\{t_{0}+1, \cdots, T\right\} \\
& h_{p, i}^{t-1}+\sum_{s \in S} f_{p, s, i}^{t}-\sum_{p \in P_{f}} \sum_{i^{\prime} \in \mathcal{W}} B_{P_{f}, p} \cdot f_{p, i, i^{\prime}}^{t}-h_{p, i}^{t}=0 \\
& \forall i \in \mathcal{M}, \forall p \in P_{r}, \forall t \epsilon\left\{t_{0}+1, \cdots, T\right\} \\
& h_{p, i}^{t}-\sum_{s \in S} f_{p, s, i}^{t}=0 \quad \forall i \in \mathcal{M}, \forall p \in P_{r}, \forall t=t_{0} .
\end{aligned}
$$

Constraints (17) and (18) are related to the storage capacity of raw material in the production facilities, as well as finished product in the distribution centers. Maximum production capacity, based on the number of installed manufacturing equipment, is calculated by Eq. (19). Also, the maximum amount of installable manufacturing equipment in each production facility is determined in Constraint (20):

$$
\begin{aligned}
& \sum_{p \in P_{f}} I S_{p} \cdot\left(h w_{p, i^{\prime}}^{t}+\sum_{i \in \mathcal{M}} \frac{1}{A_{i, i^{\prime}}} \cdot f_{p, i, i^{\prime}}^{t}\right) \leq \operatorname{ICAP}_{i^{\prime}} \cdot x_{i^{\prime}}^{t} \\
& \forall i^{\prime} \in \mathcal{W}, \forall t \epsilon \mathcal{T}, \\
& \sum_{p \in P_{r}} I S_{p} \cdot\left(h m_{p, i}^{t}+\sum_{s \in S} \frac{1}{A_{s, i}} \cdot f_{p, s, i}^{t}\right) \leq \operatorname{ICAP}_{i} \cdot x_{i}^{t}
\end{aligned}
$$

$$
\forall i \in \mathcal{M}, \forall t \epsilon \mathcal{T}
$$

$$
\begin{aligned}
& \sum_{p \in P_{f}} \sum_{i^{\prime} \in \mathcal{W}} W L_{p, i} . f_{p, i, i^{\prime}}^{t} \leq \sum_{\tau=t_{0}}^{t-1} \text { CAP.MEQ }{ }_{i}^{t, \tau} \\
& \forall i \in \mathcal{M}, \forall t \epsilon\left\{t_{0}+1, \cdots, T\right\}, \\
& \sum_{\tau=t_{0}}^{t} \operatorname{MEQ}_{i}^{t, \tau} \leq \mathrm{MEQ}_{i}^{\mathrm{MAX}} \quad \forall i \in \mathcal{M}, \forall t \epsilon \mathcal{T} .
\end{aligned}
$$

The safety stock of raw material and finished products are calculated by Constraints (21) and (22), respectively:

$$
\begin{aligned}
& h m_{p, i}^{t-1} \geq \alpha_{p} \cdot \sum_{p \in P_{f}} \sum_{i^{\prime} \in \mathcal{W}} B_{p, P_{r}} \cdot f_{p, i, i^{\prime}}^{t} \\
& \forall i \in \mathcal{M}, \forall p \in P_{r}, \forall t \epsilon\left\{t_{0}+1, \cdots, T\right\}, \\
& h w_{p, i}^{t-1} \geq \beta_{p} \cdot \sum_{c \in \mathcal{C}} f_{p, i, c}^{t} \\
& \forall i \in \mathcal{W}, \forall p \in P_{f}, \forall t \epsilon\left\{t_{0}+1, \cdots, T\right\} .
\end{aligned}
$$

Constraints (23)-(25) guarantee that only opened facilities receive or send products:

$$
\begin{aligned}
& \sum_{p \in P_{f}} \sum_{i^{\prime} \in \mathcal{W}} f_{p, i, i^{\prime}}^{t} \leq \text { BM. } x_{i}^{t-1} \\
& \forall i \in \mathcal{M}, \forall t \epsilon\left\{t_{0}+1, \cdots, T\right\}, \\
& \sum_{p \in P_{f}} \sum_{i \in \mathcal{M}} f_{p, i, i^{\prime}}^{t} \leq \text { BM. } x_{i^{\prime}}^{t-1} \\
& \forall i^{\prime} \in \mathcal{W}, \forall t \epsilon\left\{t_{0}+1, \cdots, T\right\}, \\
& \sum_{p \in P_{f}} \sum_{c \in \mathcal{C}} f_{p, i, c}^{t} \leq \text { BM. } x_{i}^{t-1} \\
& \forall i \in \mathcal{W}, \forall t \epsilon\left\{t_{0}+1, \cdots, T\right\} .
\end{aligned}
$$

Based on Constraint (26), the total number of finished products sent to a customer zone in each period is limited to the demand of that market:

$$
\sum_{i^{\prime} \in \mathcal{W}} f_{p, i^{\prime}, c}^{t} \leq D_{p, c}^{t} \quad \forall c \in \mathcal{C}, \forall p \in P_{f}, \forall t \in \mathcal{T}
$$

Constraint (27) ensures that the established facility will remain open till the end of the planning horizon:

$$
x_{i}^{t-1}-x_{i}^{t} \leq 0 \quad \forall i \in \mathcal{M} \cup \mathcal{W}, \forall t \epsilon\left\{t_{0}+1, \cdots, T\right\}
$$

Constraints (28) and (29) determine the initial value 
of cash, as well as the inventory of raw material in the first period, respectively:

$$
\begin{aligned}
& \mathrm{CASH}^{t}-\mathrm{ICASH}=0 \quad \forall t=t_{0}, \\
& \sum_{\forall i \in \mathcal{W}} h w_{p, i}^{t}-I h_{p}=0 \quad \forall p \in P_{f}, \forall t=t_{0} .
\end{aligned}
$$

Constraint (30) requires that $x_{i}^{t}$ be a binary variable. Constraints (31)-(34) restrict some variables from taking negative values. Constraint (35) attributes customer demand and the selling price of finished products to joint stochastic space:

$$
\begin{aligned}
& x_{i}^{t} \epsilon\{0,1\} \quad \forall i \in \mathcal{M} \cup \mathcal{W}, \forall t \epsilon \mathcal{T}, \\
& \mathrm{FA}^{t}, \mathrm{DEP}^{t}, \mathrm{AR}^{t}, \mathrm{AP}^{t}, \mathrm{CASH}^{t}, \mathrm{CFO}^{t}, \mathrm{TCM}^{t}, \\
& \operatorname{STD}^{t}, \mathrm{CA}^{t} \geq 0 \quad \forall t \epsilon \mathcal{T}, \\
& \mathrm{MEQ}_{i}^{t, \tau} \geq 0 \quad \forall i \in \mathcal{M}, \forall t, \tau \epsilon \mathcal{T}, \\
& f_{p, s, i}^{t}, f_{p, i, i^{\prime}}^{t}, f_{p, i^{\prime}, c}^{t} \geq 0 \\
& \forall s \in S, \forall i \in \mathcal{M}, \forall i^{\prime} \in \mathcal{W}, \forall c \in \mathcal{C}, \forall t \in \mathcal{T}, \\
& h_{P_{r}, i}^{t}, h_{P_{f}, i^{\prime}}^{t} \geq 0 \quad \forall i \in \mathcal{M}, \forall i^{\prime} \in \mathcal{W}, \forall t \epsilon \mathcal{T}, \\
& \left(D_{p, c}^{t}, \mathcal{P} \mathcal{R}_{p, c}^{t}\right) \epsilon \Omega .
\end{aligned}
$$

\section{Stochastic programming approach}

This paper applies an approach based on stochastic programming to consider the underlying uncertainty of the proposed model. Stochastic programming is a well-grounded approach to make proper decisions in a stochastic environment. Stochastic problems have some stochastic parameters with known or empirical distributions. One way to handle this problem is to work with some scenarios obtained from the population of stochastic parameters. The stochastic factors usually appear at some stage or time-period in the decision process. Accordingly, the optimal decision should be made at each stage, depending on all scenarios occurred before the current stage. To reach this, a negative correlation coefficient has been considered between the price of finished products and the associated demand [29]. The following variables are considered to be scenario dependent:

- Inventory of product $P_{f}$ in distribution center $j$ at the end of period $t: h w_{P_{f}, j}^{t, \vartheta}$;

- Flow of raw material from distribution center $j$ to customer $c$ at the beginning of period $t: f_{P_{f}, j, c}^{t, \vartheta}$;

- Total contribution margin in period $t: \mathrm{TCM}^{t, \vartheta}$;
- Cash in period $t: \mathrm{CASH}^{t, \vartheta}$;

- Cash flow from operations: $\mathrm{CFO}^{t, \vartheta}$

- Current net assets at the end of period $t: \mathrm{CA}^{t, \vartheta}$.

where $\vartheta$ is the scenario index. Accordingly, constraints (4)-(9), (14), (17), (22), (26), (28), and (29) are written for each scenario, as well.

If stochastic factors can be represented by some random variables with known or estimable probability functions, scenario-based stochastic programming requires samples from their parametric or empirical distribution functions. Several theorems have been presented to analyze multivariate distributions. However, most of them consider an identical distribution function for the multiple correlated variables [30,31]. In some cases, there are several coupled random variables with non-identical probability patterns and generating random samples for them might be of interest. Copula theory and Nataf transformation are two powerful methods introduced for this purpose $[32,33]$.

\subsection{Scenario generation method}

Stochastic programming requires some samples from stochastic parameters that are combined into the scenarios. Next, each scenario is applied to develop the final stochastic programming model. In this paper, a method, based on the Nataf transformation, is proposed to generate correlated continuous random variables. The basics of the Nataf method are given below.

Let $X=\left(x_{1}, \cdots, x_{n}\right)$ be random variables with marginal probability distribution functions, $f\left(x_{i}\right), i=$ $1,2, \cdots, n$, and correlation matrix, $\rho^{\prime}$. This vector of random variables can be transformed to a vector of multivariate normal distribution, $Y=\left(y_{1}, \cdots, y_{n}\right)^{\prime} \sim$ $N_{n}(0, \rho)$ by the following equations:

$$
\Phi\left(y_{i}\right)=F\left(x_{i}\right) \quad \forall i=1,2, \cdots, n,
$$

where $\Phi($.$) denotes cumulative normal probability func-$ tion and $F($.$) is the cumulative distribution function of$ random variables. The elements of $\rho$ are obtained by the following equations:

$$
\begin{aligned}
\rho_{i j}^{\prime} & =\int_{-\infty}^{\infty} \int_{-\infty}^{\infty}\left(\frac{x_{i}-\mu_{i}}{\sigma_{i}}\right)\left(\frac{x_{j}-\mu_{j}}{\sigma_{j}}\right) f_{x_{i} x_{j}}\left(x_{i}, x_{j}\right) d x_{i} d x_{j} \\
& =\int_{-\infty}^{\infty} \int_{-\infty}^{\infty}\left(\frac{F_{i}^{-1}\left(\Phi\left(y_{i}\right)\right)-\mu_{i}}{\sigma_{i}}\right)\left(\frac{F_{j}^{-1}\left(\Phi\left(y_{j}\right)\right)-\mu_{j}}{\sigma_{j}}\right) \\
& \varphi\left(y_{i}, y_{j}, \rho_{i j}\right) d y_{i} d y_{j}, \\
\forall i & =1,2, \cdots, n, \quad \forall j=i, i+1, \cdots, n .
\end{aligned}
$$

Therefore, the resultant multivariate normal distribu- 


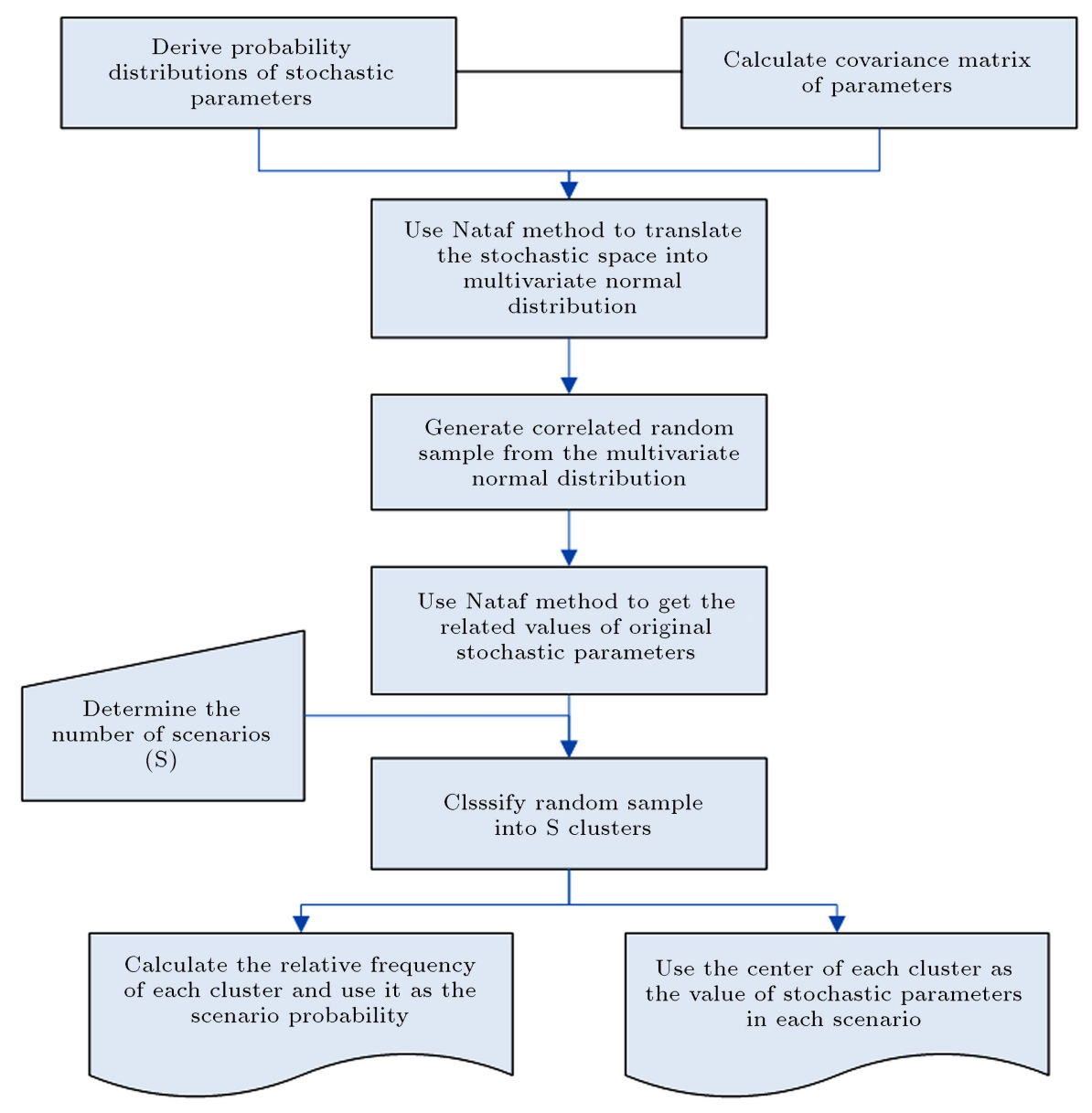

Figure 2. Method of scenario generation.

tion can be considered for sample generation. Afterwards, the normal variables are again converted to the original variables $(X)$. Figure 2 shows the proposed method of the scenario generation method proposed in this paper.

The main steps of the proposed scenario generation method can be described as follows:

Step 1. Derive marginal distribution functions for original stochastic variables and calculate their covariance matrix. This can be done by gathering historical data from each variable and conducting some non-parametric statistical hypothesis tests on goodness of fit that can evaluate and select a proper probability distribution function. Chi-square, AndersonDarling, and Kolmogorov-Smirnov tests are the main methods in attaining this goal [34];

Step 2. Apply marginal distributions and the covariance matrix in the Nataf transformation algorithm to construct a multivariate normal distribution;

Step 3. Generate samples from the correlated normal distribution; methods such as Cholesky and eigenvector decomposition could be ap- plied [34]. Such methods are available in most statistical software packages such as Minitab and SAS;

Step 4. Invert the generated normal variables to the original values using Nataf transformation equations.

\section{Computational analysis}

This section conducts some computational experiments to evaluate the performance of the proposed solution approach. Different test problems were designed in three classes, with five test problems $\left(S_{1}-S_{5}\right)$ in the small class $(S)$, three test problems $\left(M_{1}-M_{5}\right)$ in the medium class $(M)$, and six test problems $\left(L_{1}\right.$ and $\left.L_{6}\right)$ in the large class $(L)$. In the small and medium classes of the test problem, five instances, and, in the large class of the test problems, six test problems with a different number of scenarios (5-30), are generated. Table 3 illustrates the structure of the test problems. For all generated instances, the planning horizon was fixed to five periods, and the number of customers was fixed to 10. Demand and price were assumed to be uniformly distributed with known correlation, which could be 
Table 3. Structure of the test problems.

\begin{tabular}{|c|c|c|c|c|c|c|}
\hline \multicolumn{2}{|c|}{ Instances } & \multirow{2}{*}{ Suppliers } & \multirow{2}{*}{ Manufacturers } & \multirow{2}{*}{ Warehouses } & \multirow{2}{*}{ Row material } & \multirow{2}{*}{ Scenarios } \\
\hline Class & ID & & & & & \\
\hline \multirow{5}{*}{ Small } & $S_{1}$ & 2 & 2 & 1 & 10 & 5 \\
\hline & $S_{2}$ & 2 & 2 & 1 & 10 & 15 \\
\hline & $S_{3}$ & 2 & 2 & 1 & 10 & 20 \\
\hline & $S_{4}$ & 2 & 2 & 1 & 10 & 25 \\
\hline & $S_{5}$ & 2 & 2 & 1 & 10 & 30 \\
\hline \multirow{5}{*}{ Medium } & $M_{1}$ & 5 & 5 & 5 & 20 & 5 \\
\hline & $M_{2}$ & 5 & 5 & 5 & 20 & 15 \\
\hline & $M_{3}$ & 5 & 5 & 5 & 20 & 20 \\
\hline & $M_{4}$ & 5 & 5 & 5 & 20 & 25 \\
\hline & $M_{5}$ & 5 & 5 & 5 & 20 & 30 \\
\hline \multirow{6}{*}{ Large } & $L_{1}$ & 10 & 10 & 10 & 30 & 5 \\
\hline & $L_{2}$ & 10 & 10 & 10 & 30 & 10 \\
\hline & $L_{3}$ & 10 & 10 & 10 & 30 & 15 \\
\hline & $L_{4}$ & 10 & 10 & 10 & 30 & 20 \\
\hline & $L_{5}$ & 10 & 10 & 10 & 30 & 25 \\
\hline & $L_{6}$ & 10 & 10 & 10 & 30 & 30 \\
\hline
\end{tabular}

Table 4. Computational results.

\begin{tabular}{|c|c|c|c|c|c|}
\hline \multicolumn{2}{|c|}{ Instances } & \multirow{2}{*}{ Single equations } & \multirow{2}{*}{ Single variables } & \multirow{2}{*}{ Discrete variables } & \multirow{2}{*}{ CPU (s) } \\
\hline Class & ID & & & & \\
\hline \multirow{5}{*}{ Small } & $S_{1}$ & 1,351 & 1,344 & 15 & 0.206 \\
\hline & $S_{2}$ & 3,521 & 3,194 & 15 & 0.557 \\
\hline & $S_{3}$ & 4,606 & 4,119 & 15 & 0.818 \\
\hline & $S_{4}$ & 5,691 & 5,044 & 15 & 0.697 \\
\hline & $S_{5}$ & 6,776 & 5,969 & 15 & 1.746 \\
\hline \multirow{5}{*}{ Medium } & $M_{1}$ & 2,856 & 7,778 & 50 & 13.978 \\
\hline & $M_{2}$ & 6,386 & 16,228 & 50 & 103.534 \\
\hline & $M_{3}$ & 8,151 & 20,453 & 50 & 186.441 \\
\hline & $M_{4}$ & 9,916 & 24,678 & 50 & 302.192 \\
\hline & $M_{5}$ & 11,681 & 28,903 & 50 & 3897.987 \\
\hline \multirow{6}{*}{ Large } & $L_{1}$ & 5,666 & 26,668 & 100 & 56.263 \\
\hline & $L_{2}$ & 8,281 & 35,018 & 100 & 91.675 \\
\hline & $L_{3}$ & 10,896 & 43,368 & 100 & 581.896 \\
\hline & $L_{4}$ & 13,511 & 51,718 & 100 & 444.101 \\
\hline & $L_{5}$ & 16,126 & 60,068 & 100 & $>3 \mathrm{hrs}$ \\
\hline & $L_{6}$ & 18,741 & 68,418 & 100 & $>3 \mathrm{hrs}$ \\
\hline
\end{tabular}

obtained even from the slope of linear relationships (details are provided in the appendix).

These instances were solved using the CPLEX MIP solver. The CPLEX MIP solver was run on a Dual core $2.26 \mathrm{GHz}$ processor with $2 \mathrm{GHz}$ of RAM.
Computational results are shown in Table 4. From the results, we observe that in the small class of instances, the total number of extended equations varies from 1,351 to 6,776 , while the total number of variables falls between 1,344 and 5,969. For all instances in this 

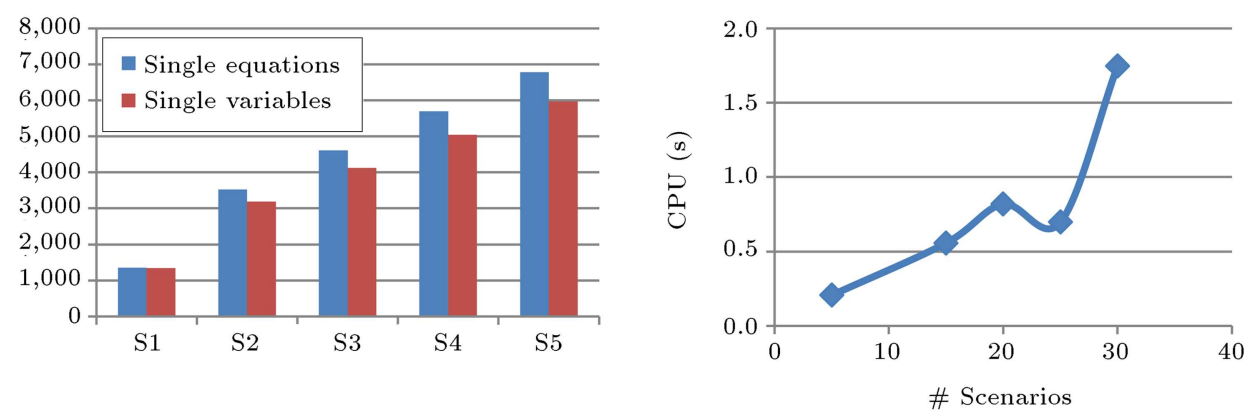

(a) Computational results for small size of instances
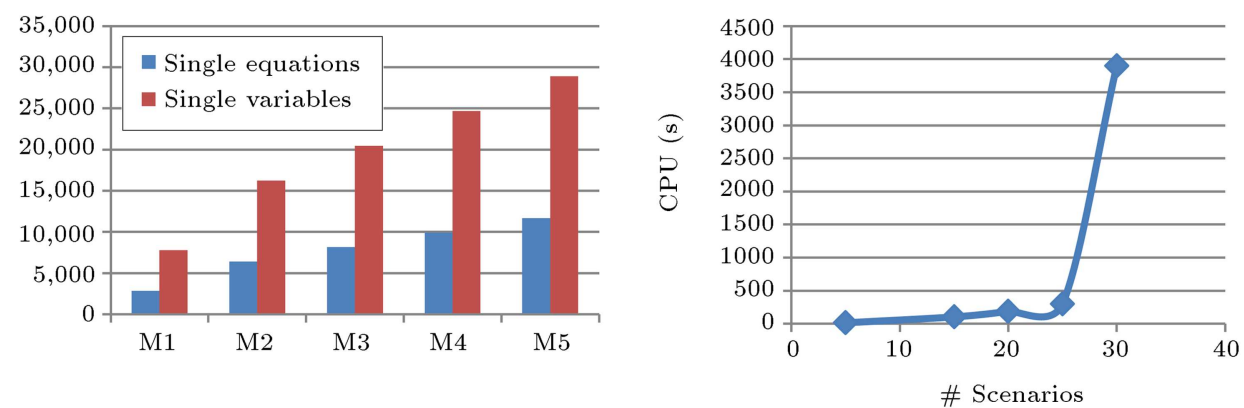

(b) Computational results for medium size of instances
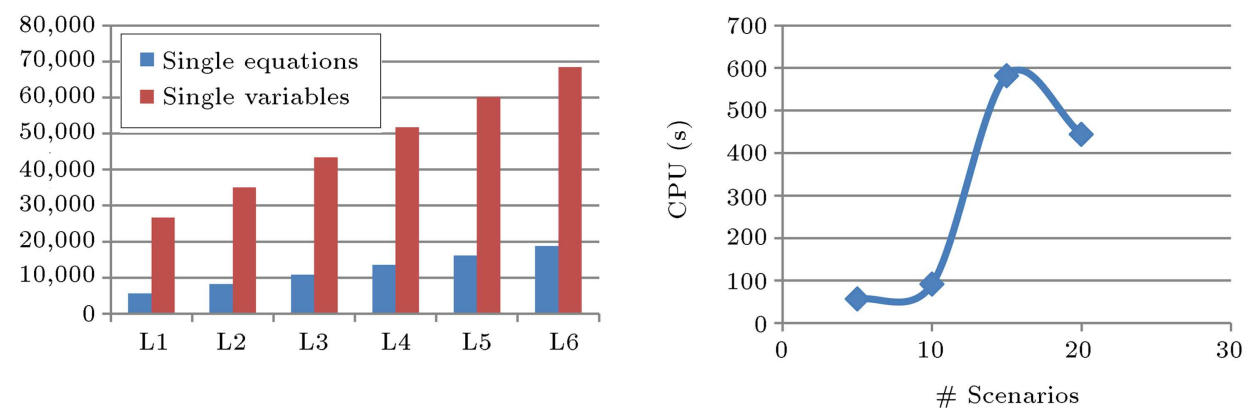

(c) Computational results for large size of instances

Figure 3. Computational results for different classes of instances.

class, the total number of discrete variables is 15 . The CPU time for these instances varies from $0.2 \mathrm{~s}$ to $1.7 \mathrm{~s}$, indicating a good performance for this class of problem (see Figure 3(a)).

In the medium class of instances, the total number of equations starts from 2,856 (M1) and reaches 11,681 (M2). Also, the number of single variables for this class of test problem varies from 7,778 to 28,903 . Furthermore, the total number of discrete variables for all generated instances in this class is 50. In this class of problems, the increasing trend in the CPU time is significant, varying from $13.9 \mathrm{~s}$ to $3897 \mathrm{~s}$ (see Figure 3(b)).

In the large class of instances, the total number of single equations exceeds 18,000 . Also, while the minimum number of discrete variables is 26,668 in instance L1, it reaches 68,418 in instance L6. The total number of discrete variables in this class of problem is 100. The CPU time is $56 \mathrm{~s}$ for the smallest test problem in this class (L1), $91 \mathrm{~s}$ for L2, $581 \mathrm{~s}$ for L3, and 444 for L4. But, the CPU time in instances $L_{5}$ and $L_{6}$ exceeds $3 \mathrm{~h}$ (see Figure $3(\mathrm{c})$ ).

Based on computational results, the adopted approach is efficient for all test problems in the small size class, all test problems in the medium size, and some test problems in the large size. But, for the large size problems with more than 40 nodes (suppliers, manufacturing facilities, distribution centers, and customers) and more than 25 scenarios, the current approach cannot find the optimum solution in a reasonable amount of time $(3 \mathrm{~h})$.

\section{Conclusion}

This paper proposes a new stochastic mixed integer linear programming model for the supply chain network problem from a value based approach. To illustrate the position of this approach in the literature and 
investigate to what extent the researchers have taken the value based concepts into consideration, an extensive review was conducted on papers in the field of supply chain network design. Based on the valuebased approach, Supply Chain (SC) configuration and planning is undertaken in such a way that the value of $\mathrm{SC}$ is maximized. Also, SC costs, sales growth, working capital, and fixed assets are supposed to be the main value drivers. Furthermore, it is assumed that customer demand and the selling price of the finished products are stochastic parameters with correlated behavior.

Also a scenario-based two-stage programming approach is developed to consider the underlying uncertainty of the proposed model. Within the scenario generation process, Nataf transformation was applied to generate correlated continuous random variables. To evaluate the performance of the developed solution approach, some numerical instances are generated and solved. The results indicate that the developed solution procedure could find a robust solution in a reasonable amount of time.

\section{References}

1. Brandenburg, M., Quantitative Models for ValueBased Supply Chain Management, Springer-Verlag Berlin Heidelberg (2013).

2. Christopher, M. and Ryals, L. "Supply chain strategy: Its impact on shareholder value", International Journal of Logistics Management, 10, pp. 1-10 (1999).

3. Hahn, G.J. and Kuhn, H. "Simultaneous investment, operations, and financial planning in supply chains: A value-based optimization approach", Intern. Journal of Production Economics, 140(2), pp. 559-69 (2012).

4. Shapiro, J., Modeling the Supply Chain, Second Ed., Thompson (2007).

5. Damodaran, A., The Little Book of Valuation - How to Value a Company, Pick a Stock, and Profit, Wiley (2011).

6. Damodaran, A., Applied Corporate Finance, 3rd Ed., Wiley (2011).

7. Rappaport, A., Creating Shareholder Value: A Guide for Managers and Investors, Second Ed., New York, Free Press (1998).

8. Aghezzaf, E. "Capacity planning and warehouse location in supply chains with uncertain demands", Journal of the Operational Research Society, 56, pp. 453-62 (2005).

9. Ambrosino, D. and Scutell, M.G. "Distribution network design : New problems and related models", European Journal of Operational Research, 165, pp. 610-24 (2005).

10. Pishvaee, M.S. and Torabi, S.A. "A possibilistic programming approach for closed-loop supply chain network design under uncertainty", Fuzzy Sets and Systems, 161(20), pp. 2668-83 (2010).

11. Christopher, M. and Ryals, L. "Supply chain strategy: Its impact on shareholder value", International Journal of Logistics Management, 10(1), pp. 1-10 (1999).

12. Walters, D. "The implications of shareholder value planning and management for logistics decision making", International Journal of Physical Distribution and Logistics Management, 29(4), pp. 240-58 (1999).

13. Lambert, D. and Pohlen, T. "Supply chain metrics", International Journal of Logistics Management, 12(1), pp. 1-19 (2001).

14. Otto, A. and Obermaier, R. "How can supply networks increase firm value? A causal framework to structure the answer", Logistics Research, 1, pp. 131-48 (2009).

15. Hahn, G.J. and Kuhn, H. "Value-based performance and risk management in supply chains: A robust optimization approach", International Journal of Production Economics, 139(1), pp. 135-44 (2012).

16. Guillén, G., Mele, F.D., Bagajewicz, M.J., Espuña, A. and Puigjaner, L. "Multiobjective supply chain design under uncertainty", Chemical Engineering Science, 60, pp. 1535-53 (2005).

17. Sabri, E.H. and Beamon, B.M. "A multi-objective approach to simultaneous strategic and operational planning in supply chain design", Omega, 28, pp. 58198 (2000).

18. Chan, Y., Carter, W.B. and Burnes, M.D. "A multipledepot, multiple-vehicle, location-routing problem with stochastically processed demands", Computers \& Operations Research, 28, pp. 803-26 (2001).

19. Melkote, S. and Daskin, M.S. "Capacitated facility location/network design problems", European Journal of Operational Research, 129, pp. 481-95 (2001).

20. Hwang, H.-S. "Design of supply-chain logistics system considering service level", Computers \& Industrial Engineering, 43, pp. 283-97 (2002).

21. Lieckens, K. and Vandaele, N. "Reverse logistics network design with stochastic lead times", Computers \& Operations Research, 34, pp. 395-416 (2007).

22. Lowe, T.J., Wendell, R.E. and Hu, G. "Screening location strategies to reduce exchange rate risk", European Journal of Operational Research, 136, pp. 573-590 (2002).

23. Miranda, P.A. and Garrido, R.A. "Incorporating inventory control decisions into a strategic distribution network design model with stochastic demand", Transportation Research Part E, 40, pp. 183-207 (2004).

24. Miranda, P.A. and Garrido, R.A. "Valid inequalities for Lagrangian relaxation in an inventory location problem with stochastic capacity", Transportation Research Part E, 44, pp. 47-65 (2008).

25. Shen, Z.M. and Qi, L. "Incorporating inventory and routing costs in strategic location models", European 
Journal of Operational Research, 179, pp. 372-89 (2007).

26. Snyder, L.V., Daskin, M.S. and Teo, C.-P. "The stochastic location model with risk pooling", European Journal of Operational Research, 179, pp. 1221-38 (2007).

27. Ommeren, J.C.W.V., Bumb, A.F. and Sleptchenko, A.V. "Locating repair shops in a stochastic environment", Computers \& Operations Research, 33, pp. 1575-94 (2006).

28. Kaplan, R.S. and Atkinson, A.A., Advanced Management Accounting, 3rd Ed., Prentice-Hal (1998).

29. Simchi-Levi, D., Kaminsky, P. and Simchi-Levi, E., Designing and Managing the Supply Chain: Concepts, Strategies \& Case Studies, McGraw-Hill (2009).

30. Johnson, N.L., Kotz, S. and Balakrishnan, N., Discrete Multivariate Distributions, Wiley New York (1997).

31. Kotz, S., Balakrishnan, N. and Johnson, N.L., Continuous Multivariate Distributions, Models And Applications, London: John Wiley \& Sons (2004).

32. Liu, P.-L. and Der Kiureghian, A. "Multivariate distribution models with prescribed marginals and covariances", Probabilistic Engineering Mechanics, 1(2), pp. 105-12 (1986).

33. Nelsen, R.B., An Introduction to Copulas, New York, Springer (1999).

34. Golub, G.H. and Van Loan, C.F., Matrix Computations, 4th Ed., Baltimore, Johns Hopkins University Press (2012).

\section{Appendix: A procedure for Nataf transformation}

\section{Parameters}

$\mathcal{P} \mathcal{R}=[p r]:$ Prices;

$D=[d]:$ Demands;

$\Phi($.$) : Cumulative distribution function of the normal$ variable.

\section{Inputs}

$F_{p r}(p r)=\frac{p r-L_{p r}}{U_{p r}-L_{p r}}:$ Cumulative distribution function of the uniform variable for the price;

$F_{d}(d)=\frac{d-L_{d}}{U_{d}-L_{d}}$ : Cumulative distribution function of the uniform variable for the demand;

$P^{\prime}$ : Correlation matrix between $\mathcal{P} \mathcal{R}$ and $D$.

\section{Nataf transformations}

Find new normally distributed variables, so that;

1. $M=0$; vector of means;
2. $P=g\left(P^{\prime}\right)$; correlation matrix, where $g($.$) is the$ function mentioned before by Eq. (37).

Generate random samples from the multivariate normal distribution; $Z \sim N(M, P)$.

Convert the generated samples to original variables using Eq. (36).

\section{Biographies}

Hossein Badri is a $\mathrm{PhD}$ degree candidate in the Department of Industrial Engineering at Amirkabir University of Technology, Tehran, Iran. His research interests are in supply chain network design, multiple criteria decision making, and combinatorial optimization methods. He is author and co-author of more than 20 technical papers in these fields.

Seyyed Mohammad Taghi Fatemi Ghomi was born in Ghom, Iran, on March 11th, 1952. He received his BS degree in Industrial Engineering from Sharif University of Technology, Tehran, Iran, in 1973, and a $\mathrm{PhD}$ degree in the same subject from the University of Bradford, England in 1980. He worked as planning and control expert in construction and cement industries, and the Organization of National Industries of Iran from 1980-1983, where he founded the Department of Industrial Training in 1981. He is currently Professor in the Department of Industrial Engineering at Amirkabir University of Technology, Tehran, Iran, where he was recognized as one of the best researchers of years 2004 and 2006, and best professor at the university in 2014. He was also recognized by the Ministry of Science and Technology as one of the best Professors of Iran for 2010. He is author and co-author of more than 350 technical papers and six books in the area of Industrial Engineering, and has supervised 133 $\mathrm{MSc}$ and $19 \mathrm{PhD}$ theses. His research and teaching interests are in stochastic activity networks, production planning, scheduling, queueing theory, statistical quality control, and time series analysis and forecasting.

Taha-Hossein Hejazi received his $\mathrm{PhD}$, MS and BS degrees in Industrial Engineering from Amirkabir University of Technology, Shahed University of Tehran, and Sadjad University of Technology, Iran, respectively. His primary research areas include quality and reliability engineering, simulation, and multiple criteria analysis. He has also written four books in areas of industrial engineering, such as discrete and continuous system simulation, quality and reliability engineering, and multiple criteria decision making. 